\section{Figuring in Friction A Pedagogical Framework for Foundational Studios}

It is a truism, perhaps, that architectural education should not merely teach tools, vocationally. Architectural education should prioritize conceptual development, interpretive skills, and critical thinking alongside calisthenic exercises in precision, craft, and rigor. The field of architecture however, continues to adopt an expanding array new mediums, predominantly computational and digital, of increasing complexity. Moreover, facility with new digital tools increasingly serves as a perquisite for entry contemporary architectural practice, presenting urgent questions and challenges for foundational architectural education. Architectural education, especially foundational pedagogy, must impart the fundamentals and simultaneously prepare students for the onset professional practice in which they will face an expanding, fragmented landscape of new architectural tools and mediums.

Critical questions for foundational pedagogy include the degree to which tool instruction and shoptalk is positioned within the studio environment. Is pedagogy strengthened by the integration of tool instruction within the studio, or should it be siloed outside in dedicated courses? Among new mediums, which best serve as vehicles for imparting design principles? Which modes of production, historically established or new and experimental, best prepare students for professional practice? Does a focused, targeted adoption of specific tools foster conceptual development, or should a wide-range of tools be sampled? Lastly, amid these questions, where can students find space to experiment, assume risk, and begin to establish their own positions?

This paper proposes a pedagogical framework for situating these questions within a foundational architecture studio and presents results from a new core curriculum at the Tulane School of Architecture, in New Orleans. A seminal foundational studio pedagogy developed a decade ago at the school is revisited and reappraised in the context of the revised curriculum. Current and past curricula share common roots and goals, but diverge in technique, method, and process. Lesson structures similar to the past curricula were adopted in the current pedagogy to facilitate systematic comparisons between approaches and make legible new outcomes. Development of core studio foundational pedagogy necessitates a clear stance on the role of tool instruction within the studio, a pressing challenge in the context of an increasingly fragmented landscape of tools, techniques, and mediums. The new pedagogy at the Tulane School of Architecture embraces this context, and positions the friction generated amidst the application of multiple tools and mediums as a primary site for architectural invention and critical development.

\section{INVENTION \& TECHNIQUE}

Since the development of projection drawing in the era of Leon Battista Alberti, architectural invention has been intrinsically coupled with the medium in which architects labor. This coupling is well-formulated by Robin Evans in a series of critical texts. In one significant example, he describes the origins and manipulations of dome of the Royal Chapel, Anet, by Philibert de l'Orme. Elaborating on discrepancies between the rational geometric forms of the dome and its floor tile pattern he writes, "It would be as crude to insist on the architect's 


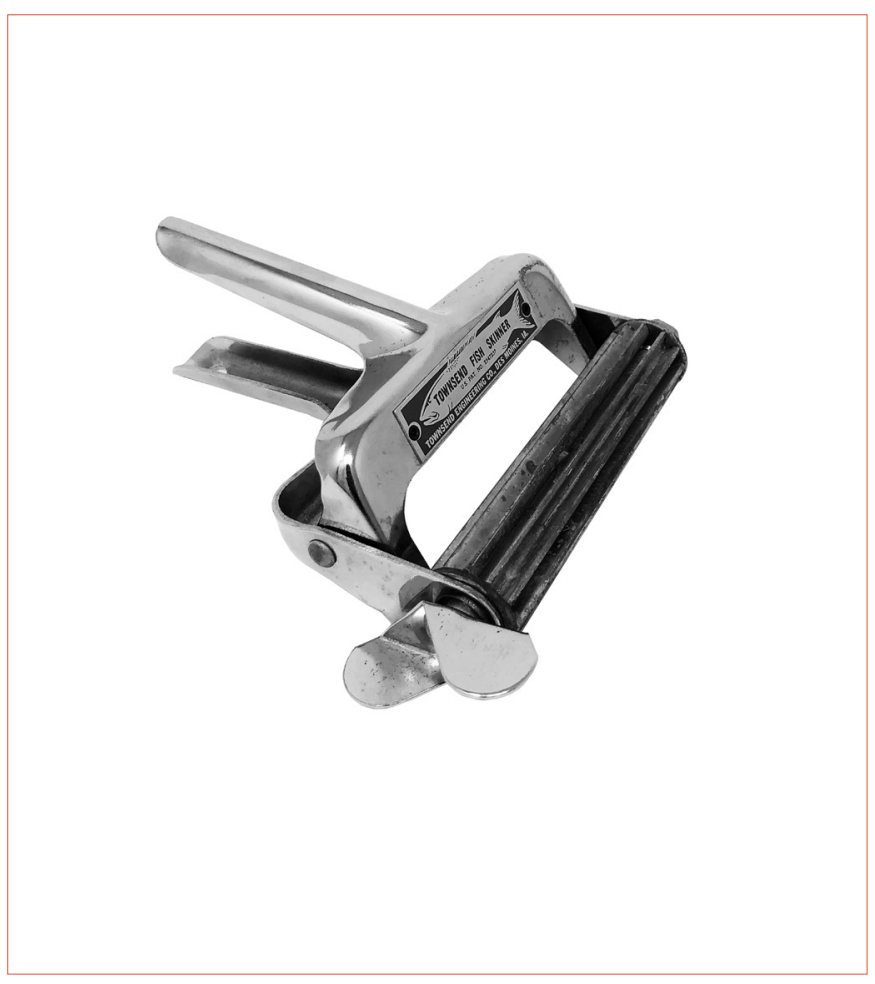

Figure 1. Fish-skinner, a typical object of study in core studio.

unfettered imagination as the true source of forms, as it would to portray the drawing technique alone as the fount of formal invention. The point is that the imagination and the technique [work] well together, the one enlarging the other." [1] Here, Evans is referring specifically to the technique of orthographic projection as the drawing technique for designing the Royal Chapel, the technology of de l'Orme's era. Without the technique of orthographic projection, the geometric manipulations within the Royal Chapel would be unimaginable.

In our present era, orthographic projection remains essential, but modes of drawing production have greatly expanded. Computational software tools automate and quicken formerly intensive drawing tasks. The circles inscribed on the dome of the Royal Chapel, for example, can now be arrayed and projected within a matter of seconds. Variants, iterations, and alternatives options can quickly follow, dramatically expanding possibilities for production. Stan Allen describes the computer as a "superpowerful drawing machine," rather than a radical paradigm shift in modes of the architectural production. [2] Others, such as John May, position the computer as an image processor, in which the former mediums of drawing and photography are supplanted by a new digital medium and cease to meaningfully exist as formerly defined. [3] The pedagogical framework established for the core curriculum at the Tulane School of Architecture assumes an agnostic approach to these questions, and seeks to allow space for students to determine their own answers-or if these questions even matter at all. The pedagogy asserts instead that there remains room for play within Evans' observation that imagination and technique

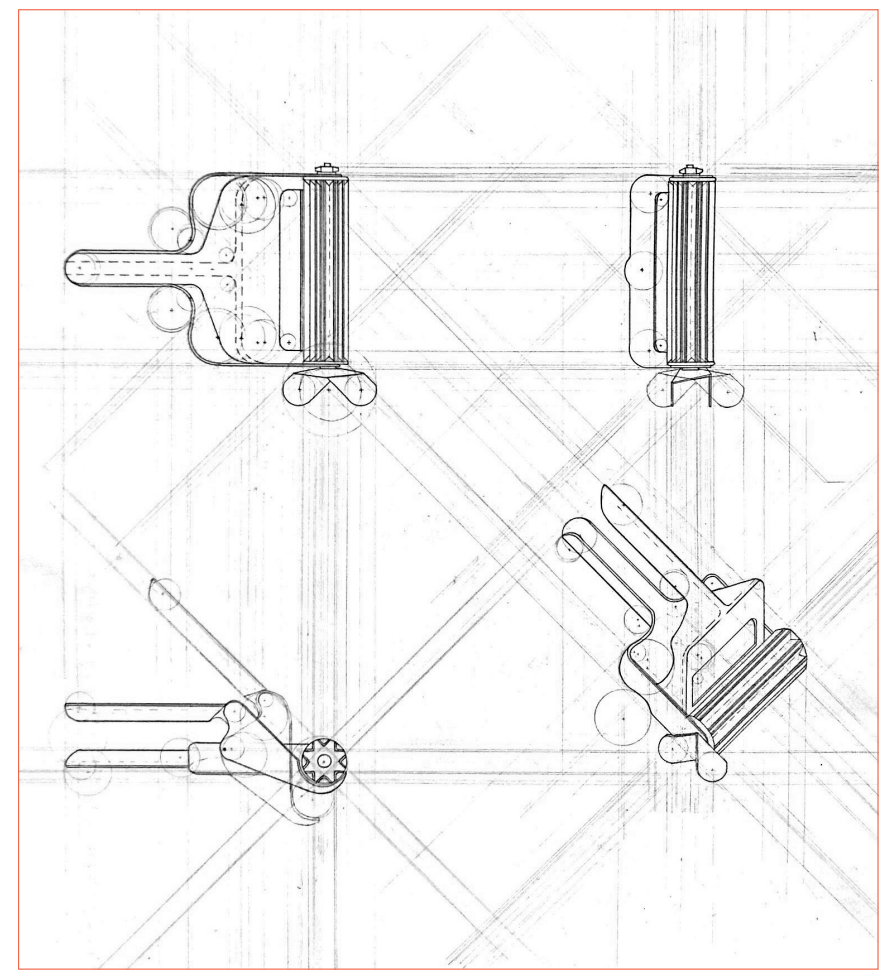

Figure 2. The fish-skinne, hand drawn orthographic projections. (Credit: Sara Bhatia)

work in concert. [4] Philibert de l'Orme may have conceived of the dome as a rational geometric deployment of sphere and inscribed circles, but the cropped projected pattern on the floor illuminates the generative friction of working between mediums and techniques.

Our present era is characterized by an expanded field of tools and techniques, and correspondingly greater possibilities for friction. Adopting and prioritizing a single technique or a single mode of production risks locating students too singularly within the current diversity of production tools and mediums. At the same time, subjecting students to a firehose of technologies, softwares, techniques risks inhibit depth and development of ideas and focus. In professional practice, friction is often framed as a problem within architectural production. There has been much discussion of interoperability among platforms, integrated BIM, and seamless project delivery workflows. [5] While there exist legitimate reasons for mitigating and minimizing friction in professional practice, correctly situated friction can be a productive agent, generating important space for invention within the discipline.

\section{FIGURE IT IN}

A decade ago, a foundational studio at the Tulane School of Architecture, developed by Associate Professor of Architecture Tiffany Lin, introduced digital collage as a means of transforming hand-drawings quickly and intuitively to generate new narratives for subsequent design exercises. [6] A deliberately prescribed sequence 


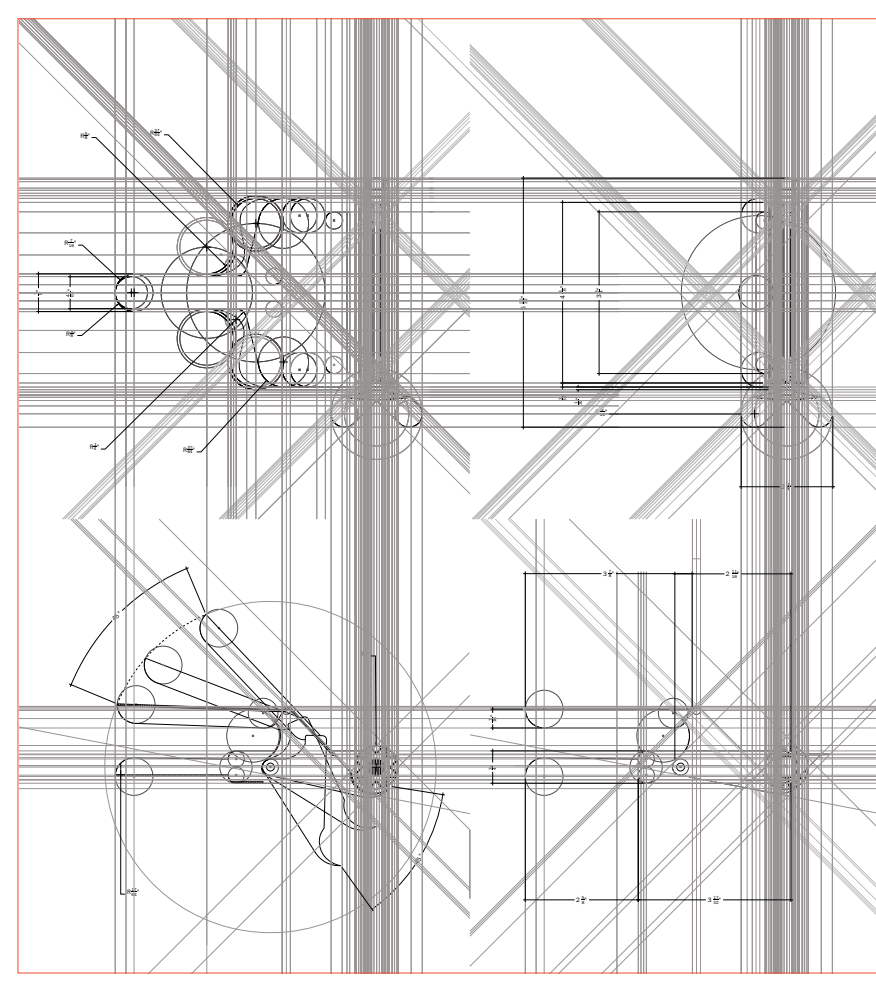

Figure 3. The fish-skinne, digitally drawn orthographic projections, (Credit: Sara Bhatia)

of projects began with an examination of mechanical devices as tactile artifacts to be analyzed and recorded. Objects were distributed or acquired by students for study in drawing and modeling. These devices consisted of handheld tools, physically operable. Tools were typically vintage, both draw from industrial, mechanical geometries rather than the more typically sinuously formed contemporary consumer products. Additionally, a degree of historical remove and corresponding unfamiliarity helped prompt looking and seeing anew. Potato-ricers, fish-skinners, and radish-dicers were explored through precision hardline hand-drawings, analog palimpsests and explicit registrations of student labors.

The objects were redrafted into Exquisite Objects through traditional tools of architecture, namely hardline hand drawing, photography, and image collage. The drawing and imaging of the tools lead to the development a series of spatial investigations in the form of "tool cases" physical models that both held the object and exhibited its mechanical properties. Drawings were again reworked through image collage, amplifying tactile qualities and interpretive depth. Photographic manipulation of the tool drawings prompted a series of spatial investigations through physical models which were again photographed and served as the subject of digital reinterpretation.

Throughout, a series of lessons sequentially introduced a new techniques, starting with hand drawing and progressing to digital image production techniques. These studies paved the way for the introduction of imaginative rescaling, inhabitation, and ultimately a set of formal principles that served as the conceptual pivot to the design

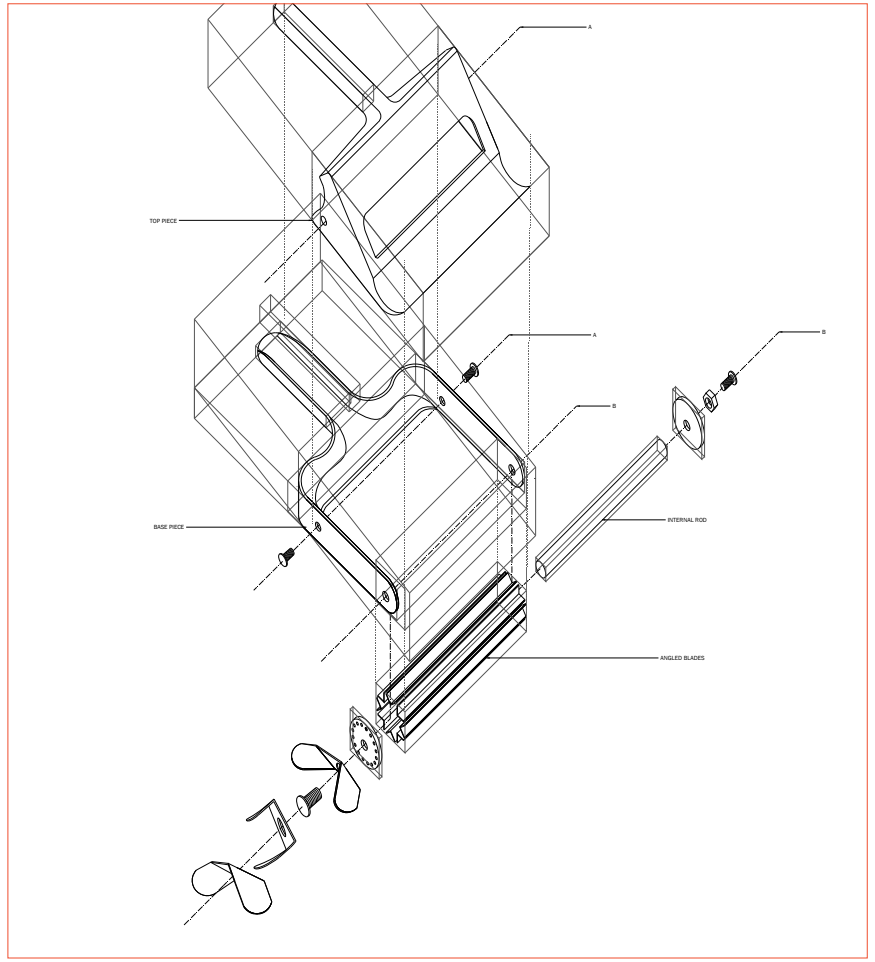

Figure 4. The fish-skinne, digitally drawn exploded axonometric. (Credit: Sara Bhatia)

of a small building. The tools were embraced to explore three-dimensional form in a two-dimensional drawing, and four-dimensional (time-based) motion in a three-dimensional modeling. Tools, in all definitions of the word, were introduced as a means of distinguishing between a tool's operational functions and its formal possibilities. Both are at once open to various interpretations, but also rooted concrete, foundational principles.

\section{NEW FRICTIONS}

The revised foundational pedagogy similarly adopts mechanical tools as objects of study, within a similar lesson arc, but introduces a nonlinear approach to digital and analog modes of production. The new curriculum situates student work strategically in the space between mediums, between freehand sketching and descriptive geometry, between physical models and digital models, between orthographic projection and computational procedures, such as boolean operations.

Additionally, strategies for graphical layering of information are incorporated from the onset. Notations and annotations are introduced as both graphic devices and strategies for illustrating interpretive depth, as an analogue to hand drawings's traditional tactile relationship with the paper. The trace of process is no longer a byproduct of a single medium but subject to control, to be constructed and composed, pushing the limitations of one medium while drawing facility from another. Fragmentation of tools and mediums needn't 


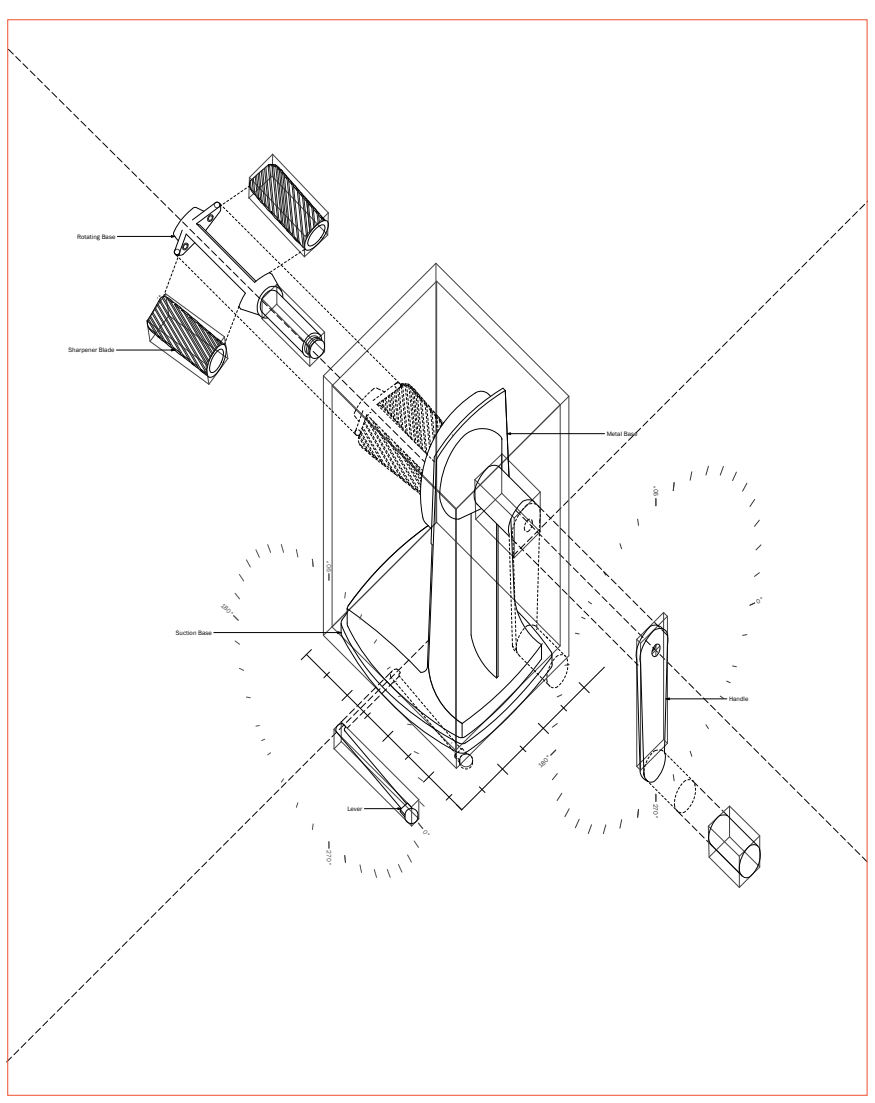

Figure 5. Pencil Sharpener, digitally drawn exploded axonometric. (Credit: Andreea Dan)

correspond to a fragmentation of process and development. Rather, the friction among tools and mediums establishes stable territory for production and development in a context in which tools, techniques, and mediums are themselves unstable.

The initial exercises of the new curriculum, like the former curriculum, began with a hand-drafted analysis of a tool, but quickly transitioned to projective geometry studies developed in digital drawing and modeling. The process was no longer linear. Instead, students were encouraged to move between analog and digital processes strategically. Notations and annotations were applied as both graphic devices and additional layering of information to generate interpretive depth-the "grit" that analogue drawings traditionally offerconstruction lines, smudges, and affectation of the hand. The trace of process is no longer an artifact of the actual process, but something to be controlled, constructed and composed. Students learned to test ideas as physical models and digital models within the same week, revealing the limitations of one medium, while expanding facility in another.

\section{SITES OF PRODUCTIVE FRICTION}

The new core pedagogy positions friction generated amidst multifaceted workflows as a primary site for architectural invention and critical development. Not all frictions are productive, of course. The friction generated by buggy computer software, for example, is typically not productive. The productive frictions located at the heart of the Tulane School of Architecture core pedagogy can be distilled to three primary categories. The first productive friction occurs between mediums. Mediums include graphite applied to fine printmaking paper, vector-based graphics plotted on coated bond paper, raster-based graphics similarly plotted, cardboard, chipboard cut by laser cutter, 3D printed polylactide (PLA) thermoplastic, and LCD screen projections. The second friction identified is between tools. These include vector line drawing, digital 3D modeling, image manipulation software, hardline analog drawing, pencil for free-hand sketching, analog sketch models, cutting mats and X-Acto knifes, and more. The third productive friction is between historical and contemporary practices. These include descriptive geometry, operational language (such as "boolean"), systems procedural logic, strategies for formal hierarchy, and image production types. Production based in the tools of contemporary practice alone risks locating our already atemporal approach to history production at a yet farther remove. Avoid contemporary tools, on the other hand, risks separating students from forms of critical thinking and craft that are instrumental to future development. The alternative, one in which productive friction can occur, is to embrace both at once.

\section{FRICTIONAL METHODOLOGIES}

In lessons and exercises, students were asked to consider what is lost and what is gained by transitioning work across mediums. Hand drawing for example, enables a tactile relationship with the paper, facilitating careful thought in each drawing and an unambiguous relationship with scale, that the computer cannot reproduce. Moreover, hand drawing has a centuries long history in the discipline, a history that still informs current practice. Computers have nonetheless become standard in architecture because they offer the ability to rapidly iterate new designs, to automate tedious work, and to create new types of representation. Hand drafting a given state of a mechanically operable object for example, requires a lengthly drawing process. Digital drafting however, can enable dozens of states with a few mouse clicks. The computer also enables iterative testing of line weights without redrawing, new possibilities for dashed line-types, automated hatching, and other graphic devices. Throughout, students were tasked with exploiting the strengths of computer drawing without losing sight of the rigor developed in hand drawing.

Lessons and exercises also borrowed heavily from the concept of the diptych. A diptych is defined as an image created in two parts, typically displayed through two conjoined panels. Diptychs are storytelling devices with origins dating to antiquity. The etymology of the word comes from the Greek root "dis", meaning "two," and "ptykhe," meaning "fold." Diptychs have been used throughout history and across multiple cultures, often to communicate religious narratives. It is also a widely used device in Modern Art, perhaps the most famous example of which is Andy Warhol's 1962 Marilyn Diptych. Diptychs locate meaning and ideas not as two parallel concepts but situated in between two parts, neither telling the entire story alone. Likewise, 


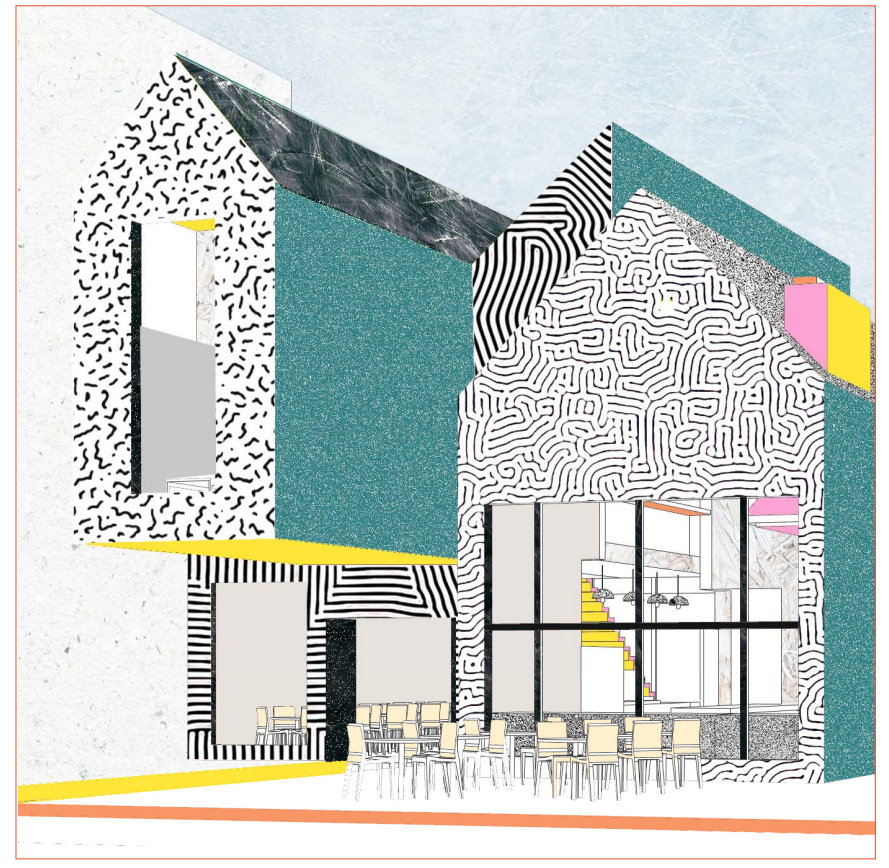

Figure 6. Bakery/restaurant facade(s), designed and drawn with a multifaceted digital and analog workflo . (Credit: Andreea Dan)

students were tasked with pairing presentation boards presenting representations developed in multiple tools and mediums. Drawing boards developed with analog and digital tools presented neither identical images, nor a completely different drawing. Rather, they presented work rooted common architectural criteria, such as scale, view orientation, and/or composition, but also stood alone as two unique boards, showcasing developments in various tools and mediums.

\section{CONCLUSION}

The approach outlined in this paper locates a new architectural pedagogy at the intersection of multifaceted analog and digital workflows. The frictions that arise within architecture's expanded field of tools, mediums, and traditions are not obstacles, but sites of opportunity. The contemporary fragmentation of tools and mediums needn't correspond to a fragmentation of process and development. Rather, the friction among tools and mediums establishes new possibilities for pedagogy and practice. Foundational pedagogy can be described as an exercise in defining, distilling, and imparting fundamentals of while simultaneously equipping students with the instruments necessary for advancing beyond the fundamentals. Correspondingly, architectural education must teach the tools and instruments of design. Individually, these tools and medium can be prescribed and instructed. Yet it is the friction generated amidst the application of multiple tools and instruments that is the site of invention. It is the space in-between that generates new knowledge, and in which intuition, ideas, and architecture operate.

\section{Notes}

1. Robin, Evans. "Translations from Drawing to Building." (1997).

2. Tehrani, Nader, and Allen, Stan. "Stan Allen." BOMB 123 (2013): 56-64.

3. May, John. "Everything is Already an Image." Log 40 (2017): 14-15.

4. Norman, Carrie. "A Drawing Forged in Two and Three Dimensions." Proceedings of the ACSA 106th Annual Meeting. Washington, D.C.: Association of Collegiate Schools of Architecture, 2019.

5. Modesitt, Adam. "Mashup and Assemblage in Digital Workflows: The Role of Integrated Software Platforms in the Production of Architecture." Architectural Design 87, no. 3 (2017): 34-41.

6. Lin, Tiffany. "Figure it in." Journal of Architectural Education 65, no. 2 (2012): 59-68. 